Pacific Journal of Mathematics

FAMILIES OF PARALLELS ASSOCIATED WITH SETS 


\title{
FAMILIES OF PARALLELS ASSOCIATED WITH SETS
}

\author{
E. E. Robkin and F. A. VAlentine
}

There exist sets $S$ in Euclidean space $E_{n}$ which have an interesting association with a family $\mathscr{P}$ of parallel lines. For instance $S$ and $\mathscr{P}$ may be so related that each point of $S$ lies on a member of $\mathscr{P}$ which intersects $S$ in either a line segment or a point. There exist compact sets $S \subset E_{2}$ such that every finite collection of points in $S$ is contained in some collection of parallel lines each of which intersects $S$ in a single point, and yet no infinite family $\mathscr{P}$ of parallel lines exists having the same property and covering $S$. This paper contains a theorem which enables one to determine the existence of a family of parallel lines each of which intersects $S$ in a line segment or point and which as a family covers $S$.

Secondly we show that the points, the closed line segments, the closed convex triangular regions, and the closed convex sets bounded by parallelograms are the only compact convex sets $B$ in $E_{2}$ which have the following property. If $A$ is a closed connected set disjoint from $B$ and if every 3 or fewer points of $A$ lie on parallel lines intersecting $B$, then $A$ is covered by a family of parallel lines each of which intersects $B$.

Finally, we obtain a theorem of Krasnoselskii type. Intuitively, this may be stated as follows. Suppose $S$ is a compact set in $E_{n}$ and suppose there exists a plane $H$ such that every $n$ points of $\mathbf{S}$ can see $H$ via $S$ along parallel lines. Then all the points of $S$ can see $H$ via $S$ along parallel lines.

The above results appear in Theorems $3,2,1$ in that order. The appendix at the end contains the theorems of Helly, Krasnosel'skii and other results used. Furthermore, the reader is recommended to consult the compendium "Helly's theorem and its relatives" by Danzer, Klee and Grünbaum [1]. In order to proceed logically we adopt the following notations.

Notation. If $S$ is a set in $n$-dimensional Euclidean space $E_{n}$, then closure of $S=\operatorname{cl} S$, interior of $S=\operatorname{int} S$, boundary of $S=b d S$, convex hull of $S=\operatorname{conv} S$. If $x \in E_{n}, y \in E_{n}, x \neq y$, then $L(x, y)=$ line containing $x$ and $y, x y=$ closed segment joining $x$ and $y$, intv $x y=$ relative interior of the segment $x y, R(x, y)=$ ray having $x$ as endpoint and containing $y$. The empty set is indicated by 0 and the origin of $E_{n}$ by $\varnothing$. Set union, intersection and difference are denoted by $\cup, \cap$ and $\sim$ respectively.

Received July 6, 1964. The preparation of this paper was sponsored (in part) by the National Science Foundation Grants GP 1368 and 1988. 
Parallels.

THEOREM 1. Let $S$ be a closed set in n-dimensional Euclidean space $E_{n}$.

(a) Suppose there exists a hyperplane $H$ such that $S \cap H$ is compact.

(b) Suppose for each integer $s$ such that $1 \leqq s \leqq n$ and for each set of distinct points $x_{1}, x_{2}, \cdots, x_{s}$ in $S \sim H$ there exist points $y_{1}, y_{2}, \cdots, y_{s}$ in $H$ such that $x_{i} y_{i} \subset S(i=1, \cdots, s)$ and such that $x_{1} y_{1}, x_{2} y_{2}, \cdots, x_{s} y_{s}$ are parallel. (The $y_{1}, \cdots, y_{s}$ need not be distinct.)

Then there exists a family $\mathscr{P}$ of parallel lines such that for each point $x \in S \sim H$ there exists a point $y \in H$ such that $x y \subset S$ and such that the line $L(x, y)$ belongs to $\mathscr{P}$.

Proof. To each $x \in S \sim H$, let $C(x)$ denote the union of all lines $L(x, y)$ where $y \in H$ such that $x y \subset S$. Choose a point $\varnothing$ as origin in $E_{n}$ with $\varnothing \notin H$, and let $D(x)$ be that translate of $C(x)$ so that $x$ goes to $\varnothing$. Define $M(x)$ as follows, when $x \in S \sim H$,

$$
M(x) \equiv \operatorname{conv}(H \cap D(x)) .
$$

Since $H \cap D(x) \neq 0$, we must have $M(x) \neq 0$ for each $x \in S \sim H$. Hypothesis (b) implies that every $n$ or fewer members of the collection $\{M(x), x \in S \sim H\}$ have a point in common. Since the dimension of $H$ is $n-1$, and since the members of $\{M(x), x \in S \sim H\}$ are compact convex sets in $H$, Helly's theorem [2] (see Appendix) for $(n-1)$ dimensional space implies

$$
\bigcap_{x \in S \sim H} M(x) \neq 0 .
$$

Since $\varnothing \notin H$, condition (1) implies there exists a line $L$ through $\varnothing$ such that $L \cap M(x) \neq 0$ for each $x \in S \sim H$. We let $\mathscr{P}$ denote the family of all lines parallel to $L$ and intersecting $S$. We will prove that $\mathscr{P}$ has the desired property stated in the theorem. To do this choose a point $x \in S \sim H$, and let $L(x)$ be the line through $x$ parallel to $L$. Let $L(x) \cap H \equiv y$ and suppose $x y \not \subset S$. Since $S$ is closed then there exists a point $u \in \operatorname{intv} x y$ and a closed solid sphere $U(u, r)$ with center $u$ and radius $r$ such that $S \cap U(u, r)=0$. Let $U(v, t)$ be a closed solid sphere with center $v \in x u$ and radius $t$ with $t<r$. Define $K(v)$ as follows,

$$
K(v) \equiv \operatorname{conv}[U(u, r) \cup U(v, t)], v \in x u .
$$

Since $S$ is closed, and since $K(u) \cap S=0, K(x) \cap S \neq 0$, there exists a point $w \in x u$ nearest to $u$ such that $S \cap b d K(w) \neq 0, S \cap \operatorname{int} K(w)=0$. Choose a point $z$ such that 


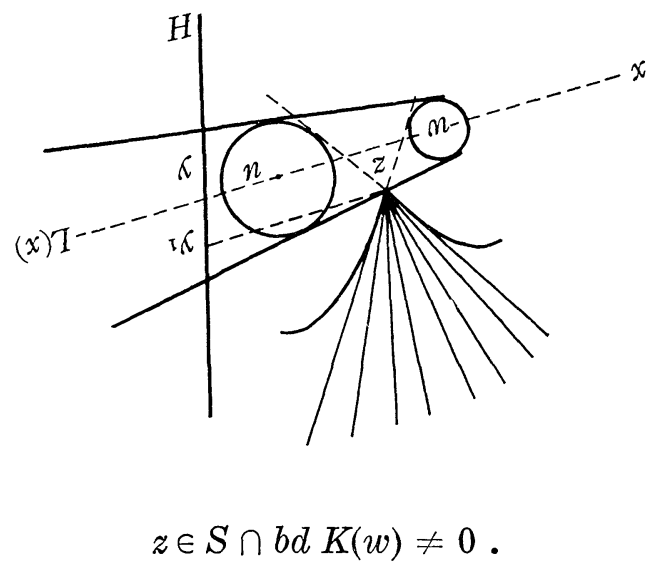

Clearly $D(z)$ contains no line $L\left(z, y_{1}\right)$ parallel to the line $L(x)$ with $y_{1} \in M(z)$. This contradicts the statement following (1). Hence $x y \subset S$, and Theorem 1 has been proved.

In order to express the next theorem easily the following concepts are used.

Definition 1. A set $A$ has the parallel property $P(m)$ relative to a set $B$ if every $m$ or fewer points of $A$ all lie on a family of parallel lines each of which intersects $B$.

A set $A$ has the parallel property $P(A)$ relative to $B$ if all the points of $A$ lie on a family $\mathscr{P}$ of parallel lines each of which intersects $B$.

THEOREM 2. Let $B$ be a compact convex set in the Euclidean plane $E_{2}$. Suppose that each closed connected set $A$ in $E_{2}$ which is disjoint from $B$ and which has property $P(3)$ relative to $B$ also has the property $P(A)$ relative to $B$.

Then $B$ is either a point, a closed line segment, a closed set bounded by a triangle or a closed set bounded by a parallelogram.

Proof. Since this theorem characterizes the sets $B$, the proof consists of two parts.

Let $\mathscr{A}=\{A\}$ denote the collection of all those closed connected sets $A$ which are disjoint from $B$ and which have property $P(3)$ relative to $B$. First, suppose $B$ is either a point, a closed line segment, a closed triangular region or a closed region bounded by a parallelogram.

Case 1. Suppose $b d B$ is a parallelogram with consecutive vertices $a_{1}, a_{2}, a_{3}, a_{4}$. The four lines determined by the four edges of $B$ divide 
the plane $E_{2}$ into nine parts. Let $V\left(a_{i}\right)(i=1,2,3,4)$ denote the unbounded open $V$-shaped region abutting $B$ at $a_{i}(i=1,2,3,4)$, and let $V\left(a_{1}, a_{2}\right)$ be the closed unbounded region abutting $B$ along $a_{1} a_{2}$, etc. Since $A \in \mathscr{A}$ is connected and disjoint from $B$, if $A \cap V\left(a_{i}\right) \neq 0$, $A \cap V\left(a_{i+1}\right) \neq 0,\left(i=1,2,3,4 ; a_{5}=a_{1}\right)$, the set $A$ would not have the property $P(2)$ relative to $B$. Hence, it would not have property $P(3)$ relative to $B$. Therefore, we may relabel the vertices of $B$ so that

$$
A \subset V\left(a_{1}, a_{2}\right) \cup V\left(a_{2}\right) \cup V\left(a_{2}, a_{3}\right) .
$$

For $x \in A$, let $C(x)$ denote the union of all rays emanating from $x$ which intersect $B$, and let $D(x)$ be that translate of $C(x)$ which sends $x$ to the origin $\varnothing$ of $E_{2}$. Since $B$ is compact and convex, and since $x \notin B$, the set $C(x)$, and hence $D(x)$, is a closed convex cone which is not all of $E_{2}$. Let $C$ be the unit circle with center at $\varnothing$ so that $C \equiv[x:\|x\|=1]$.

Define $M(x)$ as follows,

$$
C \cap D(x) \equiv M(x), x \in A .
$$

Consider the collection of sets

$$
\mathscr{C} \equiv\{M(x), x \in A\} \text {. }
$$

Property $P(3)$ implies that there exists a semicircular arc $C_{1}$ of $C$ such that every two members of $\mathscr{C}$ have a non-empty connected intersection with $C_{1}$. To see this observe that if in (2) we have $A \cap V\left(a_{2}\right) \neq 0$, then for each point $x \in A \cap V\left(a_{2}\right)$ we have a connected intersection

$$
M(x) \cap M\left(x_{1}\right) \cap M\left(x_{2}\right) \neq 0
$$

for every pair of points $x_{1}, x_{2}$ in $A$. If $A \cap V\left(a_{2}\right)=0$ then either $A \subset V\left(a_{1}, a_{2}\right)$ or $A \subset V\left(a_{2}, a_{3}\right)$, and the above italicized statement is also still true. (It is instructive to observe that condition $P(2)$ does not suffice to imply the above italicized sentence.) We may now apply Helly's theorem [2] (see Appendix) to the set $\mathscr{C}$ to yield the existence of a paint $u \in M(x)$ for all $M \in \mathscr{M}$. Let $L$ be the line determined by $\varnothing$ and $u$. For each $x \in A$, let $L(x)$ denote the line through $x$ parallel to $L$. The above facts imply that $L(x) \cap B \neq 0, x \in A$, by virtue of the definition of $C(x)$. Hence $A$ has property $P(A)$ relative to $B$.

Case 2. Suppose $B$ is a closed set bounded by a triangle with vertices $a_{1}, a_{2}, a_{3}$. As argued in case 1 , we may relabel the vertices so that

$$
A \subset V\left(a_{1}\right) \cup V\left(a_{1}, a_{2}\right)
$$


if $A \in \mathscr{A}$, where $V\left(a_{1}\right)$ is the open $V$-shaped region abutting $B$ at $a_{1}$, and where $V\left(a_{1}, a_{2}\right)$ similarly abuts $B$ along $a_{1} a_{2}$. The rest of the proof is exactly the same as Case 1 .

Case 3. Suppose $B$ is a closed segment $a_{1} a_{2}$. If $A \in \mathscr{A}$, then $A$ either lies on $L\left(a_{1}, a_{2}\right)$ or in one of the open half-spaces bounded by $L\left(a_{1}, a_{2}\right)$. The proof is either trivial or exactly the same as Case 1 , or, for that matter, as Theorem 1.

Case 4. If $B$ is a point, then $A$ must lie on a line through $B$, and the conclusion is trivial.

This completes the first part of the proof.

(I) Secondly, to complete the characterization, suppose $B$ is a compact convex set which is neither a point, a line segment, a triangular region or a set bounded by a parallelogram.

We will prove that for such a set $B$ there exists a closed connected set $A$, disjoint from $B$, which has property $P(3)$ relative to $B$, and which does not have property $P(A)$ relative to $B$. In order to construct $A$ we use the familiar concept of "exposed point."

DEFINITION 2. A point $x$ in the boundary of a convex set $S \subset E_{2}$ is an exposed point of $S$ if there exists a line $L$ of support to $S$ at $x$ such that $S \cap L=x$.

To construct $A$, let $x_{1} x_{2}$ be a diameter of the set $B$ described in the italicized statement (I). The points $x_{1}, x_{2}$ are exposed points of $B$ since the line $L_{i}$ through $x_{i}(i=1,2)$ and perpendicular to $x_{1} x_{2}$ is such that $L_{i} \cap B=x_{i}(i=1,2)$. Let $H$ be one of the open half-planes bounded by $L\left(x_{1}, x_{2}\right)$ such that $H \cap B \neq 0$, since $B \not \subset L\left(x_{1}, x_{2}\right)$. It is a well-known elementary fact that $B$ contains at least one exposed point in $H$. If $B$ contains one and only one exposed point in each of the open half-planes $H$ and $E_{2} \sim$ cl $H$, then $B$ is a quadrilateral. (We have excluded the case of a parallelogram, here.) On the other hand, if $B$ contains only one exposed point in $H$ and none in $E_{2} \sim \operatorname{cl} H$ then $B$ is bounded by a triangle, which is also excluded here. For the moment, suppose $A$ contains at least two exposed points $x_{3}$ and $x_{4}$ in $H$. Without loss of generality, suppose $x_{1}, x_{2}, x_{4}, x_{3}$ occur on $b d B$ in that order as illustrated in Figure 2. (In Fig. 2, the dotted lines and curves, except for the points $x_{1}, x_{2}, x_{3}, x_{4}$ miss $B$.)

Let $L\left(x_{i}\right)(i=3,4)$ be two lines such that $B \cap L\left(x_{i}\right) \equiv x_{i}$. Observe that

$$
\operatorname{conv}\left(x_{1} \cup x_{3} \cup x_{4} \cup x_{2}\right) \subset B \text {. }
$$

There exist points $x_{i j}(i, j=1,2,3,4, i<j)$ such that 


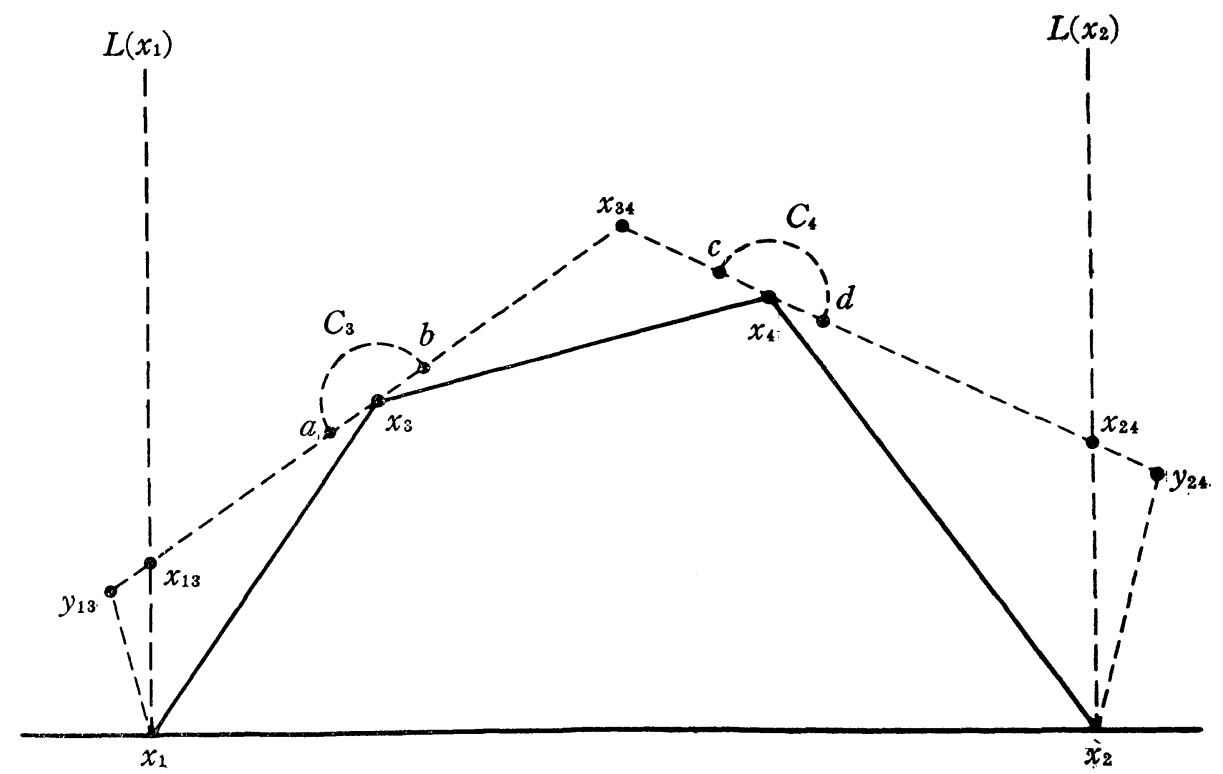

Fig. 2.

$$
x_{i j} \equiv L\left(x_{i}\right) \cap L\left(x_{j}\right)(i<j, i, j=1,2,3,4,(i, j) \neq(1,2)) .
$$

To construct the set $A$, illustrated in Figure 2, extend the segment $x_{34} x_{13}$ to $x_{34} y_{13}$ and $x_{34} x_{24}$ to $x_{34} y_{24}$ so that

$$
\begin{aligned}
& L\left(x_{2}, y_{24}\right) \cap L\left(x_{1}, x_{3}\right) \cap H \neq 0 \\
& L\left(x_{1}, y_{13}\right) \cap L\left(x_{2}, x_{4}\right) \cap H \neq 0 .
\end{aligned}
$$

Recall that $x_{3} \in H, x_{4} \in H$. Furthermore, replace a segment $a b$ of $y_{13} x_{34}$ with midpoint $x_{3}$ by a semicircular are $C_{3}$ with endpoints $a$ and $b$ and with $C_{3} \cap B=0$. Introduce a corresponding arc $C_{4}$ at $x_{4}$ (see Fig. 2). The set $A$ defined as follows

$$
A \equiv\left(y_{13} a\right) \cup C_{3} \cup\left(b x_{34}\right) \cup\left(x_{34} c\right) \cup C_{4} \cup\left(d y_{24}\right)
$$

is illustrated in Figure 2. We may choose the arcs $C_{3}$ and $C_{4}$ sufficiently small so that $A$ clearly has property $P(3)$. To see this observe first that $A \sim C_{4}$ has property $P(2)$. Hence, to see that $A$ has property $P(3)$ one merely has to demonstrate that if $x \in C_{4}, y \in A, z \in A$, the triple $\{x, y, z\}$ has property $P(3)$ relative to $B$. However, clearly the set $A$ does not have property $P(A)$ relative to $B$. This completes the proof when $B$ in statement (I) has two exposed points in $H$. The only case remaining in this part of the proof is that in which $B$ is a quadrilateral which is not a parallelogram. So to complete the proof suppose $B$ is such a quadrilateral. In this case there must exist some two vertices of $B$, say $x_{1}$ and $x_{2}$, such that the other two vertices $x_{3}$ and 
$x_{4}$ of $B$ are interior to a strip bounded by two parallel line $L\left(x_{1}\right)$ and $L\left(x_{2}\right)$ at $x_{1}$ and $x_{2}$ respectively such that $x_{3}$ and $x_{4}$ lie on the same side of $L\left(x_{1}, x_{2}\right)$. Hence, we have a situation which is essentially the same as in Figure 2 (perpendicularity was not essential), and the same construction can be carried out to yield a set $A$ having property $P(3)$ relative to $B$ but not $P(A)$. This completes the proof.

There exist further results related to property $P(m)$ and these will be presented in a subsequent paper. It should be mentioned that if in the hypothesis of Theorem 2 we replace $P(3)$ by $P(2)$ then $B$ must be either a point or a line segment. Also it is easy to prove that if $B$ is a compact strictly convex body in $E_{2}$ and if $m$ is a prescribed integer, there exists a compact connected set $A$ which is disjoint from $B$, which has property $P(m)$ relative to $B$ but which does not have property $P(A)$ relative to $B$.

THEOREM 3. Let $S$ be a closed connected set in the Euclidean plane $E_{2}$. Suppose there exist two points $a$ and $b$ in $S$ such that the following holds. If $x_{1}$ and $x_{2}$ are points in $S$ then there exist some two parallel lines, denoted by $L_{1}$ and $L_{2}$, such that $L_{i} \cap S=x_{i}$ and such that $L_{i} \cap a b \neq 0(i=1,2)$.

Then there exists a family $\mathscr{P}$ of parallel lines in $E_{2}$ such that each point of $S$ is contained in a member of $\mathscr{P}$ which intersects $S$ in either a line segment or a point.

Proof. If $x \in S$, by hypothesis there exists a line $L(x)$ through $x$ such that

$$
S \cap L(x)=x, a b \cap L(x) \neq 0 .
$$

For $x \in S$, let $C(x)$ denote the union of all possible lines $L(x)$ satisfying (4). We will prove first that $C(x)$ is a two-napped cone, each nappe of which is convex, although it need not be closed. To prove this, we consider two cases.

First, suppose $x \notin a b$. Suppose $L_{1}(x), L_{2}(x)$ are two lines in $C(x)$ through $x$. Choose an arbitrary line $L(x)$ through $x$ such that $L(x)$ intersects $a b$ between $a b \cap L_{1}(x)$ and $a b \cap L_{2}(x)$. We will show that $L(x) \cap S=x$, so that $L(x) \subset C(x)$. The proof is indirect. Suppose a point $y$ exists such that $y \in S \cap L(x), y \neq x$. By hypothesis, there exists a line $L(y)$ through $y$ such that $S \cap L(y)=y, a b \cap L(y) \neq 0$. (See Fig. 3. In this figure, the dotted lines, except for the points $x$ and $y$, miss the set $S$.) Since $L(y) \cap \operatorname{intv} a b \neq 0, L(x) \cap \operatorname{intv} a b \neq 0$, it is a simple matter to verify that the deletion from $E_{2} \sim S$ of an appropriate ray of $L(y)$ together with an appropriate ray from $L_{1}(x)$ or $L_{2}(x)$ separates the plane into two disjoint open parts, one of which 


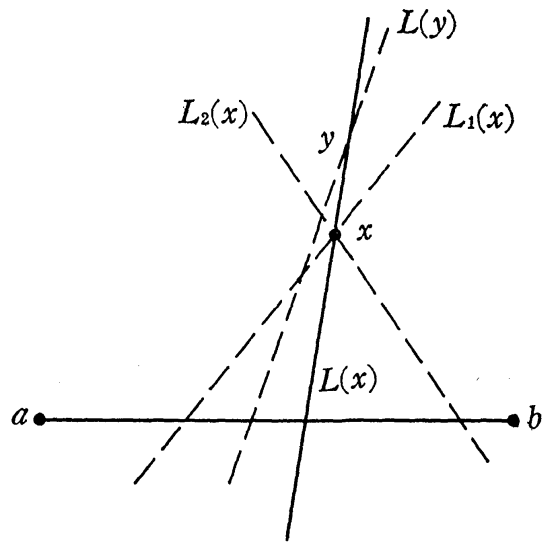

$x \notin a b$

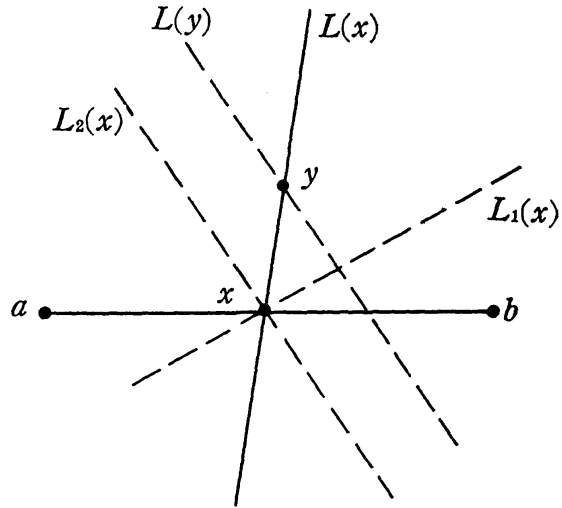

$x \in a b$

Fig. 3.

contains $a$ and the other of which contains $b$. However, this violates the fact that $S$ is connected. Hence, we have a contradiction so that $S \cap L(x)=x$. Hence, when $x \notin a b$, the set $C(x)$ is a two-napped cone, each nappe of which is convex.

Secondly, suppose $x \in S \cap a b$. The proof follows the same pattern as in the case $x \notin a b$, (see Fig. 3). The obvious details are made selfevident there.

To complete the proof, choose an origin $\varnothing$ in $E_{2}$ so that $\varnothing \notin L(a, b)$. Let $D(x)$ be that translate of $C(x)$ so that $x$ goes to $\varnothing$. Consider the collection $\mathscr{L}$, defined as follows

$$
\mathscr{L} \equiv\{M(x) \equiv L(a, b) \cap \mathrm{cl} D(x), x \in S\} .
$$

We have shown that every two members of $\mathscr{C}$ have at least one point in common. Furthermore, if $x \notin L(a, b)$, then $M(x)$ is a compact interval. If $x \in a b$ the set $M(x)$ may be unbounded (although closed and convex), however, this will cause no difficulty because if $S \subset a b$, then $\bigcap_{x \in S} M(x) \neq 0$ follows trivially, and if $S \not \subset a b$ Helly's theorem [2] can be used to yield the existence of a point $u$ such that

$$
u \in \bigcap_{x \in S} M(x) \text {. }
$$

Let $L$ be the line determined by the two points $u$ and $\varnothing$, since $\varnothing \neq u$. Let $\mathscr{P}$ denote the set of all lines in $E_{2}$ which are parallel to $L$ and which intersect $S$. We will prove that $\mathscr{P}$ is a family as described in Theorem 3, and the proof is indirect. To do this, for $x \in S$ let $L(x)$ denote that line through $x$ which belongs to $\mathscr{P}$ so that $L(x)$ and $L$ 
are parallel. Suppose a point $x \in S$ exists such that $S \cap L(x)$ is not connected. Since $S$ is closed if $S \cap L(x)$ is not connected there exist points $c$ and $d$ in $L(x)$ such that $c \in S, d \in S, c \neq d, S \cap$ intv $c d=0$. The segment $c d$ is usually called a cross-cut of the complement of $S$. Since $S$ is connected, there exists a component $K$ of the complement of $S$ such that the removal of intv $c d$ from $K$ yields two disjoint parts of $K$, at least one of which is bounded (see Fig. 4) which we denote by $K_{1}$.

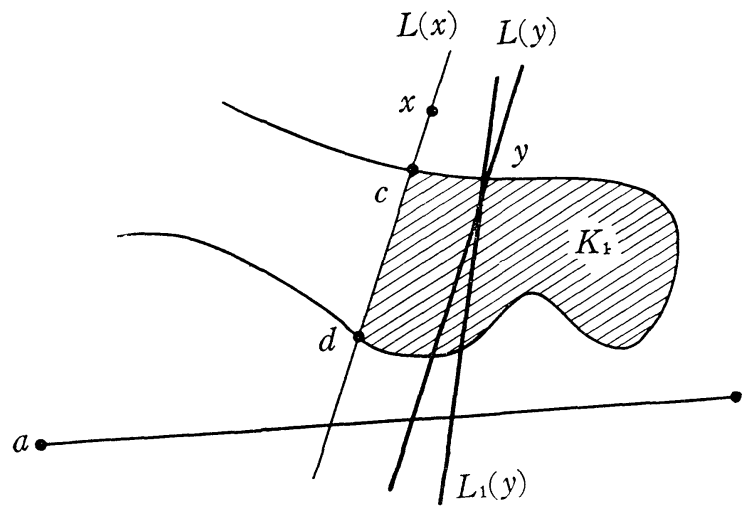

Fig. 4.

There exist points $y \in b d K_{1}$ with $y \notin L(c, d)$, sufficiently close to $L(x)$ such that the $L(y) \in \mathscr{P}$ parallel to $L(x)$ intersects $K_{1}$ and also intersects $b d K$ in a nonconnected set. Since $\operatorname{cl} C(y)$ is the closure of a nonempty two-napped cone, each nappe of which is convex, there exists a line $L_{1}(y) \subset C(y)$ through $y$, sufficiently close to $L(y)$ (in terms of angles), such that $L_{1}(y) \cap S \neq y$, a contradiction (see Fig. 4). Hence, we have proved that $\mathscr{P}$ is a desired family, and the proof is complete.

The hypotheses of Theorem 3 do not imply that a family $\mathscr{P}$ necessarily exists such that each $x \in S$ is contained in a member of $\mathscr{P}$ which intersects $S$ in just the point $x$. In fact the following is true.

There exists a compact set $S \subset E_{2}$ such that every finite collection of points $x_{1}, x_{2}, \cdots, x_{n}$ in $S$ is contained in some collection of parallel lines $L_{1}, L_{2}, \cdots, L_{n}$ such that $L_{i} \cap S=x_{i}(i=1, \cdots, n)$, and yet no family $\mathscr{P}$ of parallel lines exist such that each point of $S$ is con-

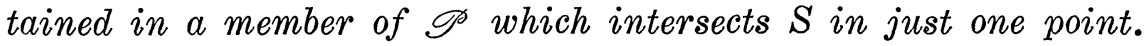

We exhibit such a set $S$ as follows.

EXAMPLE. Let $\left(x_{i}, y_{i}\right)$ denote rectangular coordinates of a point $p_{i}$ in $E_{2}$. We define the sequence of points $\left\{p_{i}, i=1,2, \cdots\right\}$ in $E_{2}$ as follows, 


$$
\begin{aligned}
\left(x_{2 n-1}, y_{2 n-1}\right) & \equiv\left(\frac{1}{2 n-1}, 0\right), \\
\left(x_{2 n}, y_{2 n}\right) & \equiv\left(\frac{1}{2 n-1}, \frac{1}{2 n-1}\right),
\end{aligned}
$$

so that $\left(x_{1}, y_{1}\right)=(1,0),\left(x_{2}, y_{2}\right)=(1,1),\left(x_{3}, y_{3}\right)=(1 / 3,0)$, etc. Define $S$ as follows

$$
S \equiv \operatorname{cl} \bigcup_{i} p_{i} p_{i+1}
$$

so that $S$ is the increasing limit of a sequence of zig-zag polygonal paths. Beginning at $(1,0)$, the odd segments are vertical, and the even segments have finite positive slope. Observe that

$$
\lim _{n \rightarrow \infty} \frac{y_{2 n-2}-y_{2 n-1}}{x_{2 n-2}-x_{2 n-1}}=\lim _{n \rightarrow \infty} \frac{2 n-1}{2}=\infty
$$

so that the even segments have slopes approaching $\infty$. It is a simple matter to verify this set $S$ has the property described in the above italicized statement because of (5) and because $x_{2 n}=y_{2 n}(n=1,2, \cdots)$.

For the concluding result we need the following concepts used by Horn and Valentine [4].

Definition 3. The set $B$ is a set of visibility for a set $S$ in $E_{n}$ if for each point $x \in S$ there exists some point $y \in B$ such that $x y \subset S$.

Definition 4. A set $S \subset E_{n}$ is said to be an $L_{2}$ set if each pair of points in $S$ can be joined by a polygonal arc consisting of at most two line segments.

Horn and Valentine [4] proved that a simply connected compact $L_{2}$ set in $E_{2}$ is expressible as the union of convex sets every two of which have a point in common. No simple characterization of nonsimply connected compact $L_{2}$ sets has ever been given. The following theorem is a step in that direction.

Theorem 4. Let $S$ be a compact $L_{2}$ set in $E_{n}$ (see Definition 3 ),

(a) then each hyperplane in $E_{n}$ has a translate which intersects $S$ in a set of visibility for $S$,

(b) also each $(n-2)$-dimensional flat is contained in a hyperplane which intersects $S$ in a set of visibility for $S$.

Proof. For each point $x \in S$, let $S(x)$ denote the set of all points $y$ such that $x y \subset S$. Also define $C(x)$ to be

$$
C(x)=\operatorname{conv} S(x) \text {. }
$$


Since $S$ is compact, the set $C(x)$ is compact. Since every two members of the collection $\{C(x), x \in S\}$ have a point in common, a theorem of Klee [6] implies that each hyperplane $H^{\prime}$ has a translate $H$ which intersects every $C(x), x \in S$. Since $S(x)$ is the union of rays having $x$ in common, the fact $H \cap C(x) \neq 0$ implies $H \cap S(x) \neq 0$. Hence, for each point $x \in S$, there exists a point $y \in H \cap S$ such that $x y \subset S$. This establishes (a). In the same manner a theorem of Horn [3] implies that each $(n-2)$-dimensional flat is contained in a hyperplane which intersects every $C(x), x \in S$, and the remainder of the proof of (b) is identical to that given for (a).

\section{Appendix}

Theorem (Helly [2]). Let $\mathscr{F}$ be a family of compact convex sets in $E_{n}$ containing at least $n+1$ members. If every $n+1$ members of $\mathscr{F}$ have a point in common, then all of the members of $\mathscr{F}$ have $a$ point in common.

TheOREM (Krasnosel'skii [5]). Let $S$ be a compact connected set in $E_{n}$. Suppose that for every $n+1$ points $x_{i} \in S(i=1, \cdots, n+1)$ there exists at least one point $y \in S$ such that $x_{i} y \subset S(i=1, \cdots, n)$. Then there exists a point $p \in S$ such that $x p \subset S$ for each point $x \in S$.

THEOREM. Let $\mathscr{F}$ be a family of bounded closed convex sets in a Euclidean space $E$. Suppose $\mathscr{F}$ contains at least $n$ members. Suppose every $n$ members of $\mathscr{F}$ have a point in common.

(Klee) Then every fiat of deficiency $n-1$ has a translate which intersects every member of $\mathscr{F}$.

(Horn) Every flat of deficiency $n$ is contained in a flat of deficiency $n-1$ which intersects every member of $\mathscr{F}$.

\section{BIBLIOGRAPHY}

1. L. Danzer, B. Grünbaum and V. Klee, Helly's theorem and its relatives, Symposium on Convexity, Proceedings of the Symposia in Pure Math. 17 Amer. Math. Soc. (1963). 2. E. Helly, Uber Mengen konvexer Körper mit gemeinschäftlichen Punkten, Jber. Deutch Math. Verein 32 (1923), 175-176.

3. Alfred Horn, Some generalizations of Helly's theorem on convex sets, Bull. Amer. Math. Soc. 55 (1949), 923-929.

4. Alfred Horn and F. A. Valentine, Some properties of $L$ sets in the plane, Duke Math. J. 16 (1949), 131-140.

5. M. A. Krasnoselskii, Sur un critère pour qu'on domain soit étoilé, Math. Sb. (61) 19 (1946), 309-310.

6. Victor Klee, On certain intersection properties of convex sets, Canad. J. Math. 3 (1951), 271-275.

7. F. A. Valentine, Convex sets, McGraw-Hill Book Co. (1964).

UNIVERSITY of CALIFornia, Los ANGELES 



\section{PACIFIC JOURNAL OF MATHEMATICS}

EDITORS

H. SAMELSON

Stanford University

Stanford, California

R. M. BLUMENTHAL

University of Washington

Seattle, Washington 98105
*J. DUGUNDJI

University of Southern California

Los Angeles, California 90007

RICHARD ARENS

University of California

Los Angeles, California 90024

\section{ASSOCIATE EDITORS}

E. F. BECKENBACH

B. H. NeumanN

F. WOLF

K. YosidA

\section{SUPPORTING INSTITUTIONS}

UNIVERSITY OF BRITISH COLUMBIA

CALIFORNIA INSTITUTE OF TECHNOLOGY

UNIVERSITY OF CALIFORNIA

MONTANA STATE UNIVERSITY

UNIVERSITY OF NEVADA

NEW MEXICO STATE UNIVERSITY

OREGON STATE UNIVERSITY

UNIVERSITY OF OREGON

OSAKA UNIVERSITY

UNIVERSITY OF SOUTHERN CALIFORNIA
STANFORD UNIVERSITY

UNIVERSITY OF TOKYO

UNIVERSITY OF UTAH

WASHINGTON STATE UNIVERSITY

UNIVERSITY OF WASHINGTON

AMERICAN MATHEMATICAL SOCIETY CHEVRON RESEARCH CORPORATION

TRW SYSTEMS

NAVAL ORDNANCE TEST STATION 


\section{Pacific Journal of Mathematics}

\section{Vol. 16, No. $1 \quad$ November, 1966}

Larry Armijo, Minimization of functions having Lipschitz continuous first

partial derivatives ............................... 1

Edward Martin Bolger and William Leonard Harkness, Some

characterizations of exponential-type distributions.............. 5

James Russell Brown, Approximation theorems for Markov operators ...... 13

Doyle Otis Cutler, Quasi-isomorphism for infinite Abelian p-groups ...... 25

Charles M. Glennie, Some identities valid in special Jordan algebras but not valid in all Jordan algebras .......................... 47

Thomas William Hungerford, A description of $\operatorname{Mult}_{i}\left(A^{1}, \cdots, A^{n}\right)$ by

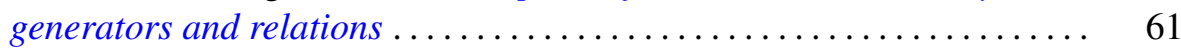

James Henry Jordan, The distribution of cubic and quintic non-residues ....

Junius Colby Kegley, Convexity with respect to Euler-Lagrange differential operators................................... 87

Tilla Weinstein, On the determination of conformal imbedding ......... 113

Paul Jacob Koosis, On the spectral analysis of bounded functions ........ 121

Jean-Pierre Kahane, On the construction of certain bounded continuous functions ................................... 129

V. V. Menon, A theorem on partitions of mass-distribution ........... 133

Ronald C. Mullin, The enumeration of Hamiltonian polygons in triangular

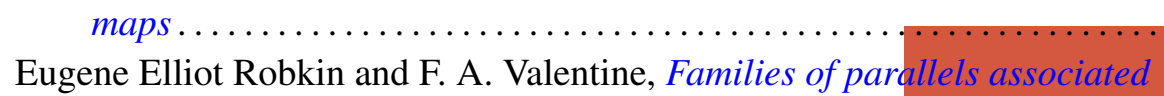

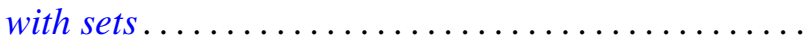

Melvin Rosenfeld, Commutative F-algebras

A. Seidenberg, Derivations and integral closure

S. Verblunsky, On the stability of the set of exponents of a Cauchy

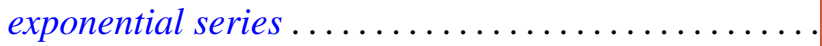

Herbert Walum, Some averages of character sums 